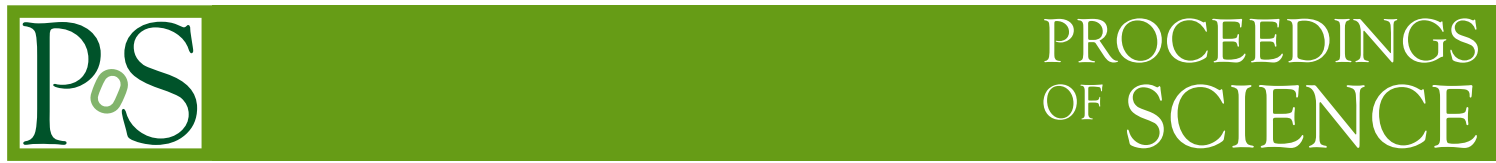

\title{
Smearing Center Vortices
}

\author{
Roman Höllwieser*12 and Michael Engelhardt ${ }^{1}$ \\ E-mail: hroman@kph.tuwien.ac.at, engel@nmsu.edu \\ ${ }^{1}$ Department of Physics, New Mexico State University, PO Box 30001, Las Cruces, NM \\ 88003-8001, USA \\ ${ }^{2}$ Institute of Atomic and Subatomic Physics, Vienna University of Technology, Operngasse 9, \\ 1040 Vienna, Austria
}

\begin{abstract}
We smear $Z(2)$ center vortices in lattice gauge configurations such as to recover thick vortices with full SU(2) Yang-Mills information. In particular, we address the problem that using $Z(2)$ configurations in conjunction with overlap (or chirally improved) fermions is problematic due to their lack of smoothness. Our method allows us to regain this smoothness and simultaneously maintain the center vortex structure. We verify our method with various gluonic and fermionic observables and find good agreement between smeared vortex configurations and full SU(2). ${ }^{\dagger}$
\end{abstract}

The 32nd International Symposium on Lattice Field Theory,

23-28 June, 2014

Columbia University New York, NY

\section{* Speaker.}

$\dagger$ The numerical simulations were performed at the Phoenix and Vienna Scientific Cluster (VSC) at VUT and the Riddler Cluster at NMSU. This research was supported by the Erwin Schrödinger Fellowship program of the Austrian Science Fund FWF ("Fonds zur Förderung der wissenschaftlichen Forschung”) under Contract No. J3425-N27 (R.H.) and the U.S. DOE through the grant DE-FG02-96ER40965 (M.E.). 


\section{Introduction}

The vortex model $[1,2,3]$ assumes that the center of the gauge group is crucial for confinement. The center degrees of freedom can be extracted from gauge field configurations by maximal center gauge (MCG) and center projection [4]. These d.o.f. are dubbed P-vortices and can be viewed as two-dimensional surfaces on the four-dimensional lattice. They are thought to approximate objects already present in configurations before the extraction step. These latter objects are called thick vortices, carry quantized magnetic center flux and are responsible for confinement according to the vortex model. The extracted P-vortex surfaces are complicated, unorientable random surfaces percolating through the lattice. These and other P-vortex properties are in good agreement with the requirements to explain confinement, which was shown both in lattice Yang-Mills theory and within a corresponding infrared effective model, see e.g. $[4,5,6,7,8,9]$. The vortex model can be applied to other infrared features of QCD not immediately related to confinement, such as the topological properties of gauge fields. In particular it was shown how the topological susceptibility present in QCD can be calculated from the extracted P-vortex surfaces $[10,11,12]$ and vortices are also able to explain chiral symmetry breaking $[13,14,15,16,17,18,19,20,21,22]$. This way the vortex model provides a unified picture for the infrared, low energy sector of QCD explaining both confinement and the chiral and topological features of the strong interaction. However, some of the properties of full QCD are obscured in the P-vortex (vortex-only) configurations, especially when it comes to topological properties in connection with fermions. In particular, we address the problem of reproducing a finite chiral condensate in center projected $(\mathrm{Z}(2))$ configurations, using overlap (and chirally-improved) Dirac operators. Low-lying eigenmodes and also zero modes are not found in these configurations; the spectra show a large eigenvalue gap for vortex-only configurations. In [17] the reason for the large gap in the vortex-only case was shown to be connected to the lack of smoothness of center-projected lattices, i.e. maximally non-trivial plaquettes - the vortex plaquettes. In that case the exact symmetry of the overlap operator is strongly field-dependent, and does not really approximate the chiral symmetry of the continuum theory.

In the present work, we want to introduce a new smearing method, in order to embed the corresponding physics from $\mathrm{Z}(2)$ vortex configurations in full $\mathrm{SU}(2)$ configurations by smoothing the thin vortices. We speculate that the infrared aspects of the QCD vacuum can be understood in terms of thick center vortices, which can be derived from thin vortex structures.

\section{Method}

The idea is to smooth out the thin vortices to regain a finite thickness, i.e. we distribute the center vortex flux of the vortex plaquettes, i.e. $\operatorname{Tr} U_{\mu v}=-1$, to several (neighboring) plaquettes. This thickens the vortices in the sense that the center flux is not restricted to a singular surface but spread out over a few lattice spacings. On the original lattice, vortex structures are only a few lattice spacings apart and smearing leads to distortions of the vortex structure. Hence, we put the vortex configuration on a finer lattice. For Z(2) gauge links, the refinement procedure can be defined straightforwardly and yields exactly the same vortex structure but on a finer lattice. The refinement procedure is illustrated in Fig. 1. We double the number of links in each direction, hence the lattice volume increases by a factor of $2^{4}=16$. If the initial link was $\mathbb{1}$, we only insert 
two $\mathbb{1}$ links; however, if the initial link has value $-\mathbb{1}$, we insert a $\mathbb{1}$ and a $-\mathbb{1}$ link in forward direction. The new link pairs are copied forward by half the initial lattice spacing in all orthogonal directions, e.g. an $\mathrm{x}$-link $U_{x}(\vec{x})=-\mathbb{1}$ at $\vec{x}=(x, y, z, t)$ gives $\tilde{U}_{x}=\mathbb{1}$ at $(x, y, z, t),(x, y+1 / 2, z, t)$, $(x, y, z+1 / 2, t), \ldots,(x, y+1 / 2, z+1 / 2, t+1 / 2)$ and $\tilde{U}_{x}=-\mathbb{1}$ at $(x+1 / 2, y, z, t),(x+1 / 2, y+$ $1 / 2, z, t),(x+1 / 2, y, z+1 / 2, t), \ldots,(x+1 / 2, y+1 / 2, z+1 / 2, t+1 / 2)$. Just as refinement gives the same vortex structure on the finer lattice, the inverse procedure, blocking, again gives the original configuration. During blocking, the copies between the coarse lattice planes are thrown away and the two refined links, e.g. $\tilde{U}_{x}(\vec{x}=(x, y, z, t))$ and $\tilde{U}_{x}(\vec{x}=(x+1 / 2, y, z, t))$, are multiplied to reproduce the original $U_{x}(\vec{x}=(x, y, z, t))=\mathbb{1} \cdot \pm \mathbb{1}= \pm \mathbb{1}$ link. On the refined lattice, however, one now has the advantage that deformations of the vortex surface within the original lattice spacing still yield the correct vortex structure after blocking.

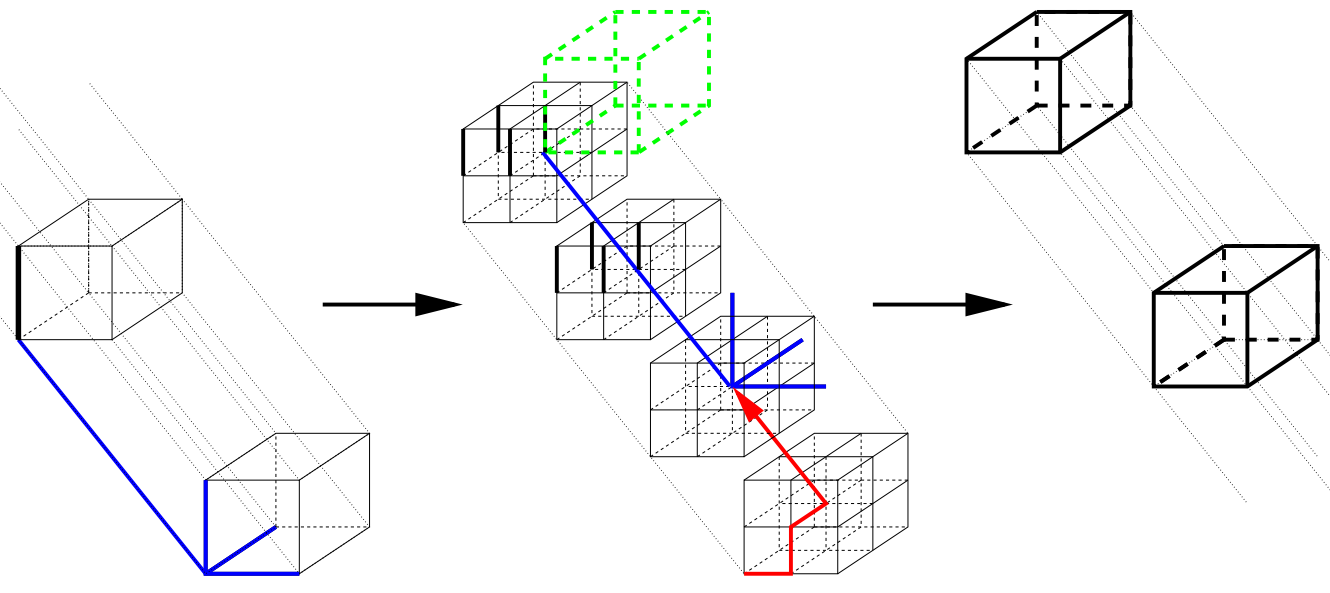

Figure 1: Refinement routine: Example of one $-\mathbb{1}$ (fat $\mathrm{z}^{-}$) link in the upper cube of the left lattice part giving eight $-\mathbb{1}$ and eight $\mathbb{1}$ links in the refined (central) lattice part. Smeared blocking procedure: After refinement and smearing the blocking is not performed starting at $(1,1,1,1)$, the lower, left corner, but half an original (i.e. one refined) lattice spacing forward in every space-time direction, i.e. $(2,2,2,2)$ on the refined lattice, indicated by the red arrow (the time direction is indicated by the fine lines connecting the space-like cubes). Without vortex smearing between steps 2 and 3 the lattices before and after the whole procedure are actually the same; with vortex smearing the re-blocked lattice gives a smeared version of the original lattice.

The goal is to distribute the center vortex flux $-\mathbb{1}$ symmetrically among the refined plaquettes making up the original vortex plaquette. Therefore we smear all four (refined) links within the original vortex plaquette by individual link rotations. In Fig. 2 we show examples of link configurations to distribute the center vortex flux $\exp i \pi=\exp -i \pi=-\mathbb{1}$ uniformly among the four refined plaquettes, each carrying one fourth of the initial center vortex flux. The uniform distribution is of course only guaranteed if we apply all link rotations of $\pm \pi / 4$ and $\pi / 2$ in the same $U(1)$ subgroup.

Depending on the vortex structure, the plotted link configurations may still cause maximally non-trivial plaquettes in directions orthogonal to the displayed plane. Hence we apply a gauge rotation to the four smeared links, multiplying them with a random $\mathrm{SU}(2)$ vector at the central point of the original vortex plaquette. Using this "2D gauge transformation" we can minimize the affected plaquettes orthogonal to the original or refined vortex plaquette. This way we eliminate maximally non-trivial plaquettes and get smooth vortex configurations on refined lattices. We finally block these smeared lattices again in order to get smooth vortex configurations on the original lattice. 

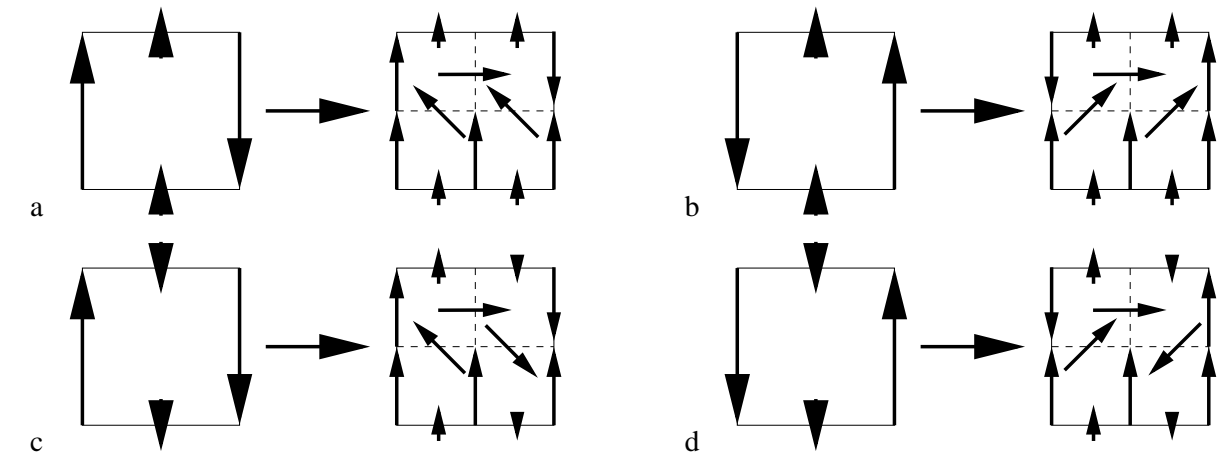

Figure 2: Examples of link configurations giving uniform center vortex flux distribution among the four refined plaquettes corresponding to the initial center vortex plaquette. The individual link rotations are given by $+\pi / 2, \pm \pi / 4$ and $\pm 3 \pi / 4$. Each refined plaquette carries one fourth of the initial center vortex flux; a),c) $\exp i \pi=-\mathbb{1}$ and $b$ ),d) $\exp -i \pi=-\mathbb{1}$. In order to minimize the orthogonal plaquettes (the ones orthogonal to the displayed plane) we apply "2D gauge transformations" (see text) at the central points of the plaquettes.

In terms of $\mathrm{Z}(2)$ lattices, we have seen that blocking is the exact inverse procedure to refinement. Hence, blocking a refined lattice exactly gives us the same links and plaquettes present in the original lattice, and therefore also the same vortex structure. Since our smearing routine only changes links at half the initial lattice spacings, i.e. links dividing the original plaquettes into four refined plaquettes, blocking trivially (the mentioned links are thrown away) restores the initial $\mathrm{Z}(2)$ link configuration and therefore the original vortex structure again. However, if we do not block the refined lattice starting at original lattice sites, but at new, refined lattice sites, more precisely one refined (half an original) lattice spacing away from original lattice sites in every space-time direction, as indicated in Fig. 1 by the red arrow, we have to multiply several smeared SU(2) links instead of $\pm \mathbb{1} \mathrm{s}$ and end up with a SU(2) instead of a Z(2) configuration. This new, blocked configuration now represents a smeared version of the original Z(2) lattice, since the smeared links are derived from the original lattice after refinement. This procedure we will call "smeared blocking" in the following, but we should note that the vortex structure extracted from the smeared blocked lattices is not exactly the same as the original vortex configuration.

\section{Results}

In order to test our method we use $1000 \mathrm{Z}(2)$ vortex configurations on $16^{4}$ lattices, derived from thermalized Luescher-Weisz SU(2) gauge field configurations on $8^{4}$ lattices at coupling $\beta=$ $3.3\left(\sigma_{\text {lat }}=0.1112 \pm 0.0017, a=0.1495 \pm 0.0012 \mathrm{fm}\right)$ after direct maximal center gauge (MCG), projection and $\mathrm{Z}(2)$ refinement.

In Fig. 3a) we display the twenty lowest-lying complex conjugate eigenvalue pairs of the overlap Dirac operator [24], for center-projected, vortex smeared and original configurations. For the spectra on the refined lattices, the eigenvalues are multiplied by a factor two, to account for the refinement effect. The refined smearing completely removes the eigenvalue gap of the center projected configurations and reproduces a finite number of (near-) zero modes. The spectrum for configurations after the smeared blocking procedure approaches the original result very well. 
a)

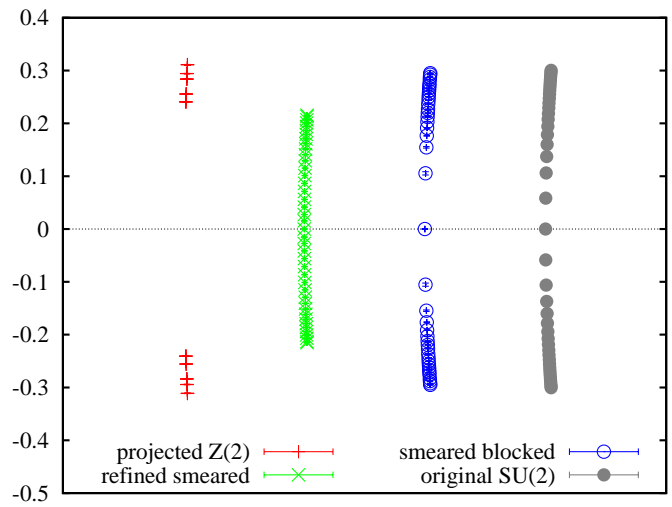

b)

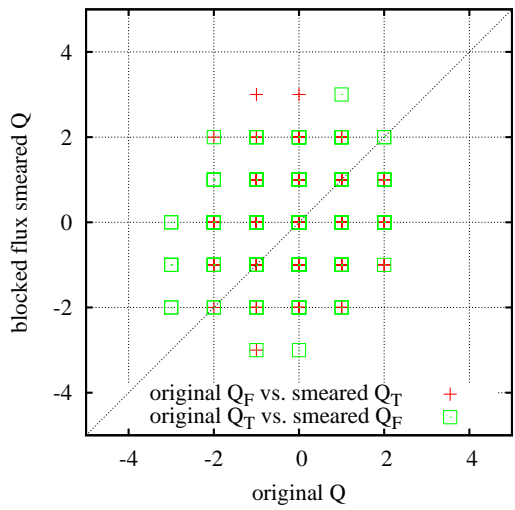

Figure 3: a) 20 lowest overlap eigenvalues for original (full) SU(2), Maximal Center Gauge projected Z(2) and vortex smeared configurations. b) Scatter plot of fermionic and gluonic topological charge correlations between original (full) vs. blocked vortex flux distribution smeared configurations.

If we want to recover the topological structure of the original (full) $\mathrm{SU}(2)$ configurations from the vortex smeared configurations, we face ambiguities: The gluonic topological charge $Q_{T}$, given by the integral of the gluonic charge density $q(x)=\frac{1}{16 \pi^{2}} \operatorname{tr}\left(\mathscr{F}_{\mu \nu} \tilde{\mathscr{F}}_{\mu \nu}\right)$, is measured after cooling or standard (e.g. LOG) smearing, which destroys the relevant vortex structures. The fermionic topological charge $Q_{F}=\operatorname{Tr}\left(\gamma_{5} D_{o v}\right)=n_{-}-n_{+}=\operatorname{ind} D_{o v}$ is given by the difference of right- and lefthanded Dirac zero modes, but it is not necessarily correlated to the vortex topological charge [15], since the vortex configurations do not represent a topological torus, as there are monopoles and Dirac strings present. During cooling or (standard) smearing, monopoles and Dirac strings are smoothed out or fall through the lattice and the toroidal topology is restored, hence $F \tilde{F}$ approaches the index topological charge. However, the vortex finding property gets lost and the vortex topological charge quickly vanishes [21]. In view of the above concerns, it is not surprising that the correlation of either fermionic $Q_{F}$ or gluonic topological charge $Q_{T}$ of the vortex smeared configurations with the original topological charge is not very good, see Fig. 3b). The vortex topological charge of original and smeared configurations is essentially the same, since we deal with identical vortex structures, however, vortex topological charge depends on the orientation of the (thick) vortex surfaces. The (thin) $\mathrm{Z}(2)$ vortices lack any information of orientation and in order to calculate the vortex topological charge, orientation is applied randomly to the vortex surfaces. Similarly, during vortex smearing, by replacing Z(2) gauge links with rotations in the SU(2) space, we automatically give the vortex surfaces a random orientation in color space, which influences the gluonic topological charge. Since these two procedures are independent, we can not expect that the smeared vortex configurations or the vortex topological charge in general give comparable results for individual configurations. However, in [10] it was shown that the vortex topological charge gives a good estimate of the topological susceptibility of the gauge field ensemble. The concepts of blocking and smoothing during vortex topological charge calculation were also discussed in [10]; the latter reduces vortex topological charge and susceptibility, since it removes short range fluctuations of the vortex structure. On our original $8^{4}$ (refined $16^{4}$ ) lattices with original lattice spacing $a \approx 0.15 \mathrm{fm}, 1-2$ (2-3) blocking steps seem appropriate during the vortex topological charge calculation in order to arrive within the range of a physical vortex thickness of $0.4 \mathrm{fm}$ [4]. 
In Fig. 4 we show the topological susceptibilities for original (full) SU(2) and smeared configurations. The first thing we note is that for our original SU(2) gauge ensemble, the topological susceptibilities from fermionic and gluonic topological charge definitions are not consistent, $\left\langle Q_{F}^{2}\right\rangle / V=(200 \mathrm{MeV})^{4}$ and $\left\langle Q_{T}^{2}\right\rangle / V=(160 \mathrm{MeV})^{4}$ (averaging cooling and LOG smearing $Q_{T}$ ), presumably caused by our small original lattice volume of about $(1.2 \mathrm{fm})^{4}$. It should be noted, however, that the vortex topological susceptibility reproduces these values with one, respectively two blocking steps, averaging over the corresponding smoothing steps. Next, we see that for the refined smeared configurations, the gluonic and vortex topological charges lead to much higher susceptibilities, caused by the artificial vortex fluctuations introduced during the refined smearing process. After blocking, however, the original results are reproduced, shown in Fig. 4b). The gluonic topological susceptibilities after cooling and (LOG) smearing the vortex smeared configurations agree with the original (full) $\mathrm{SU}(2)$ values and vortex topological charge also matches the original averages. Concerning fermionic topological susceptibility, the results are consistent with gluonic and vortex topological susceptibilities. The refined version gives $(260 \mathrm{MeV})^{4}$, after blocking we find $(160 \mathrm{MeV})^{4}$. The results show that vortices are indeed able to reproduce the topological susceptibility of full Yang-Mills theory, either via vortex topological charge or gluonic and fermionic definitions after vortex smearing.

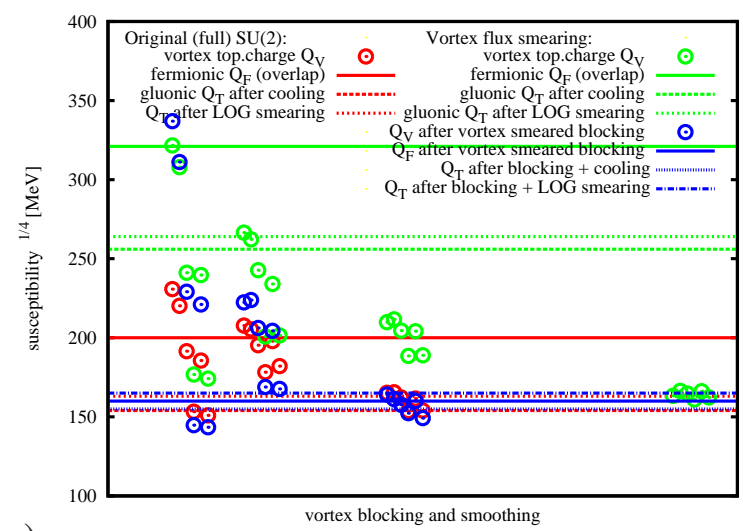

a)

Figure 4: Topological susceptibility from fermionic $Q_{F}$, gluonic $Q_{T}$ and vortex topological charge $Q_{V}$ for original (full) $\mathrm{SU}(2)$ and vortex flux distribution smeared configurations. In a) we show all blocking and smoothing steps for $Q_{V}$ (vertical groups of points correspond to 0,1,2 and 3 blocking steps, the horizontal splitting within the group indicates $0,1,2,3,4$ or 5 smoothing steps, for details see [10]), in b) we show 1-2 blocking steps for $8^{4}$ lattices, i.e. original and smeared blocked configurations, and 2-3 blocking steps for refined $\left(16^{4}\right)$ lattices, resulting in lattice spacings $a \approx 0.3-0.6 \mathrm{fm}$.

\section{Conclusions and Outlook}

We presented a method to smear Z(2) vortex configurations such as to embed vortex physics into a full $\mathrm{SU}(2)$ gauge configuration framework. The main goal was to remove the eigenvalue gap observed for overlap fermions in center-projected $\mathrm{Z}(2)$ vortex configurations. In order to maintain the original vortex structure we have to put the $\mathrm{Z}(2)$ configurations on finer lattices, where we 
can distribute the center vortex flux of the vortex plaquettes onto the refined plaquettes making up the original vortex plaquette. Our method thickens the vortices in the sense that the center flux is not restricted to a singular surface but spread out over a few lattice spacings. Besides refined smearing, we also discuss a method to block the smeared lattice back to its original size. With the various methods we can also reproduce topological properties of the original gauge fields; on vortex smeared lattices, the different definitions of topological charge, i.e. fermionic, gluonic and vortex topological charge, result in comparable susceptibilities. However, one-to-one correlations of topological charge for individual configurations are not observed. The reason is that gluonic topological charge definitions are usually applied after cooling or standard smearing, both transforming Monte Carlo configurations into smooth gauge fields without or with strongly distorted center vortex excitations. Thin center vortex gauge fields, i.e. $\mathrm{Z}(2)$ configurations, on the other hand lack any information on the orientation of thick center vortices, which is crucial for topological charge determination. During vortex smearing or vortex topological charge determination, we apply random orientations to the vortex sheets and cannot expect to reproduce the original topological charge. However, earlier results and the analysis presented here and in more detail in [25], show that vortex gauge fields reproduce the original topological charge susceptibility via vortex topological charge definition and via fermionic or gluonic definitions after the introduced vortex smearing methods.

\section{References}

[1] G. 't Hooft, Nucl. Phys. B138 (1978) 1.

[2] P. Vinciarelli, Phys. Lett. B78 (1978) 485-488.

[3] J. M. Cornwall, Nucl. Phys. B157 (1979) 392.

[4] L. Del Debbio, M. Faber, J. Greensite, and Š. Olejník, Phys. Rev. D 55 (1997) 2298-2306.

[5] K. Langfeld, H. Reinhardt, and O. Tennert, Phys. Lett. B419 (1998) 317-321.

[6] T. G. Kovacs and E. T. Tomboulis, Phys. Rev. D 57 (1998) 4054-4062.

[7] M. Engelhardt and H. Reinhardt, Nucl.Phys. $B 585$ (2000) 591-613.

[8] M. Engelhardt, M. Quandt, and H. Reinhardt, Nucl.Phys. B685 (2004) 227-248.

[9] R. Höllwieser, D. Altarawneh, and M. Engelhardt, arXiv:1411.7089 [hep-lat].

[10] R. Bertle, M. Engelhardt, and M. Faber, Phys. Rev. D 64 (2001) 074504.

[11] M. Engelhardt, Nucl.Phys. B585 (2000) 614.

[12] M. Engelhardt, Phys. Rev. D 83 (2011) 025015.

[13] P. de Forcrand and M. D'Elia, Phys. Rev. Lett. 82 (1999) 4582-4585.

[14] C. Alexandrou, P. de Forcrand, and M. D'Elia, Nucl. Phys. A663 (2000) 1031-1034.

[15] M. Engelhardt, Nucl.Phys. B638 (2002) 81-110.

[16] V. Bornyakov et al., Phys. Rev. D 77 (2008) 074507.

[17] R. Höllwieser, M. Faber, J. Greensite, U.M. Heller, and Š. Olejník, Phys. Rev. D 78 (2008) 054508.

[18] R. Höllwieser, M. Faber, U.M. Heller, arXiv:1005.1015 [hep-lat ].

[19] R. Höllwieser, M. Faber, U.M. Heller, JHEP 1106 (2011) 052.

[20] T. Schweigler, R. HÃúllwieser, M. Faber, and U. M. Heller, Phys.Rev. D87 no. 5, (2013) 054504.

[21] R. Höllwieser, M. Faber, U.M. Heller, Phys. Rev. D 86 (2012) 014513.

[22] R. Höllwieser, T. Schweigler, M. Faber and U.M. Heller, Phys.Rev. D88 (2013) 114505.

[23] R. Narayanan and H. Neuberger, Nucl. Phys. B443 (1995) 305-385.

[24] R. Höllwieser and M. Engelhardt, arXiv:1503.00016 [hep-lat] . 